\title{
Warm Hearts and Cool Heads: Uncomfortable Temperature Influences Reliance on Affect in Decision-Making
}

\author{
RHONDA HADI AND LAUREN BLOCK
}

\begin{abstract}
Can uncomfortable temperature exposure systematically influence consumers' reliance on affect in decision-making? Using a thermoregulatory framework in which individuals are motivated to maintain thermal comfort, we propose that individuals instinctively adopt a more (less) affective decision-making style in response to uncomfortable physical cold (warmth). We demonstrate that the adoption of an affective decision-making style makes individuals feel warmer (study 1) and more comfortable in response to uncomfortably cold temperature (study 2). Accordingly, individuals spontaneously rely more or less on affect when feeling uncomfortably cold or warm, respectively (study 3), which ultimately influences consequential downstream variables (e.g., willingness to pay; studies 4 and 5). This effect holds in response to both tactile (studies 3 and 4) and ambient (study 5) temperature exposure and is most exaggerated at extreme temperatures (when thermoregulatory objectives are at their strongest).
\end{abstract}

$\bar{T}$ he New York Times discovered a curious correlation across retail stores in Manhattan: the more expensive the merchandise, the colder the store temperature (Salkin 2005). Reports across the globe suggest analogous situations: Hong Kong's luxurious IFC mall is kept at a frigid 59 degrees Fahrenheit (Willett 2015), and in the United Kingdom, high-end grocery stores are "positively arctic" (Pursglove 2013). Even promotional campaigns can leave consumers shivering: Columbia Sportswear set up a mobile walk-in freezer, where consumers were encouraged to sing karaoke while testing their products (Newman 2011). These examples suggest that although temperature setting may be a methodical consideration for managers, conditions are hardly optimized for patron comfort.

While an important body of research has documented consumer responses to temperature fluctuations within a modest, comfortable range (e.g., Ijzerman and Semin 2009; Cheema and Patrick 2012), little research has looked at how consumers respond to uncomfortable temperature exposure. This distinction is not only practically relevant (as our introductory examples illustrate) but is also theoretically meaningful since the accompanying discomfort can motivate compensatory tendencies: those who feel cold seek warmth, and those who feel warm seek coolness (Zhang and Risen 2014). The limited research examining consumer responses to uncomfortable temperatures has demonstrated that cold temperatures increase the favorability of metaphorically warm stimuli (e.g., people prefer romantic movies when it is cold outside; Hong and Sun 2012). Our research moves beyond an examination of metaphorical stimuli preference to propose that uncomfortable temperature exposure can in fact influence the extent to which consumers rely on an affective decision-making process when making judgments and choices.

First, we demonstrate that use of an affective decisionmaking process can make individuals feel warmer. Despite allegorical references to "hot" versus "cold" processing systems (e.g., Metcalfe and Mischel 1999), no research has empirically examined this notion before. Further, we demonstrate that individuals instinctively alter their decisionmaking process to compensate for uncomfortable temperature exposure (relying more or less on affect to warm up or cool down, respectively). Thus, the present work makes a

Rhonda Hadi (corresponding author; rhonda.hadi@sbs.ox.ac.uk) is an associate professor of marketing at Saïd Business School, University of Oxford, Park End Street, Oxford OX1 1HP, UK. Lauren Block (lauren.block@baruch.cuny.edu) is the Lippert Professor of Marketing at Baruch College, City University of New York, One Bernard Baruch Way, New York, NY 10010. This work is based on part of the first author's doctoral dissertation. The authors would like to thank Aradhna Krishna and Andrew Stephen for their helpful comments on earlier versions of this work. The authors also thank the editor and reviewers for their constructive feedback.

JACR, volume 4, number 2. Published online January 17, 2019. http://dx.doi.org/10.1086/701820

(c) 2019 the Association for Consumer Research. All rights reserved. 2378-1815/2019/0402-0038\$10.00 
unique contribution by identifying a novel antecedent of affective processing, while simultaneously extending the literature on consumer responses to thermal conditions.

\section{THEORETICAL BACKGROUND}

\section{Consumer Responses to Temperature Variations}

Comfortable Temperature Exposure. Research examining consumer responses to temperature has generally focused on a comfortable range of thermal conditions. Notably, a stream of research suggests that comfortable sensory experiences can induce metaphorically assimilative judgements akin to semantic priming (Lakoff and Johnson 1999; Förster, Liberman, and Friedman 2007; Zhang and Li 2012). For example, exposure to comfortable (or very brief) physical warmth can activate metaphorical concepts of emotional warmth. Physical warmth (vs. cold) has led participants to judge confederates as having a "warmer" personality (Williams and Bargh 2008), induced greater social proximity, and a more relational focus (Ijzerman and Semin 2009), and has led to decreased product distance perceptions and increased product valuations (Zwebner, Lee, and Goldenberg 2013).

Other work has looked at how temperature variations can affect decision-making. Drawing on resource depletion theory, Cheema and Patrick (2012) demonstrate that modestly warm (vs. cool) ambient temperatures can lead individuals to rely on system 1 processing (characterized by effortless, heuristic-based, and quick judgments; Evans 2008) and accordingly perform worse on complex cognitive tasks (e.g., difficult gambles, proofreading tasks). While the current work also explores the influence of temperature on decision-making, our exploration differs in two critical ways. First, while Cheema and Patrick (2012) explore the effect of temperature on system 1 versus system 2 processing, we explore how temperature influences consumer reliance on affect. While dual processing accounts sometimes refer to emotions under the category of system 1 processing, contemporary research suggests that reliance on affect can often be part of a deliberate and controlled process (Feldman Barrett, Ochsner, and Gross 2007; Keren and Schul 2009), and affective decision-making taps into a separate system of judgment with its own characteristics and consequences (Chang and Pham 2013). Second, the current work also extends the aforementioned research by focusing on how consumer decision-making shifts in response to uncomfortable temperatures. As we discuss next, uncomfortable temperatures tend to elicit a distinct pattern of consumer responses.
Uncomfortable Temperature Exposure. The literature distinguishes between how individuals make decisions in response to comfortable temperatures and to those that are outside their comfort zone-in other words, in temperatures that may feel "too cold" or "too warm." This distinction is crucial, as only thermally experienced discomfort elicits goal-directed responses and motivates compensatory tendencies: uncomfortably cold individuals seek warmth (e.g., a warm drink) and uncomfortably warm individuals seek coolness (e.g., a cold drink; Panksepp 1998; Zhang and Risen 2014). This reasoning is consistent with literature suggesting that while mere semantic activation may lead to assimilative judgments, goal activation should lead to contrast effects (Förstercet al. 2007; Sela and Shiv 2009; Zhang and Risen 2014).

Interestingly, a growing body of literature suggests that uncomfortable temperature does not only lead consumers to compensate by consuming materially warm or cool stimuli but can also drive consumer preference for metaphorically cold or warm stimuli. Hong and Sun (2012) were among the first scholars to demonstrate that cold individuals show greater preference for metaphorically warm products. Using online rental records, as well as experimental lab studies, these authors demonstrate that physical coldness increases preference and willingness to pay for romantic movies (Hong and Sun 2012). Likewise, an uncomfortably cold state can lead to greater preference for interpersonal products and activities (Lee, Rotman, and Perkins 2014; Zhang and Risen 2014), nostalgic music (Zhou et al. 2012), and metaphorically "warm" advertisements (i.e., those conveying happiness or love; Bruno, Melnyk, and Volckner 2016). Zhang and Risen (2014) document the crucial role that motivation plays in such contrasting preferences by empirically demonstrating that uncomfortable thermal temperature contains a motivational element that activates the goal to "undo" the discomfort or restore comfort. The underlying logic is that consuming metaphorically warm stimuli can make individuals feel warmer and thus help compensate for the experience of excess physical cold. Some researchers have even objectively measured the efficacy of such complementarity, showing that exposure to metaphorically warm concepts (e.g., social inclusion or environmental friendliness) can increase participants' skin temperature (Ijzerman et al. 2012; Taufik, Bolderdijk, and Steg 2014). Taken together, these studies provide supportive evidence that uncomfortable thermal states lead to contrasting stimuli preference.

However, no research has looked at how a consumer's decision-making process itself-regardless of displayed choice 
or preference - might be used to compensate for uncomfortable temperature exposure. This is surprising given the wellestablished semantic associations that link affective mental processes with warm thermal qualities. In the behavioral sciences specifically, "warm" processes refer to those involving affect, feelings, and desires, while "cool" processes refer to those involving calculative cognition, linear progression, and critical analysis (Metcalfe and Mischel 1999; Peters et al. 2006). In fact, the notion of "warm" affect dates as far back as Aristotle, who argued that internal equilibrium is afforded via the interplay of the "hot heart" and "cold brain" (Gross 1995). This symbolism is further epitomized in everyday language: a person who decides quickly and irrationally is "hotheaded," while a person restraining their emotion stays "cool as a cucumber." Thus, historical perspectives, laymen's expressions, and academic terminology all reinforce the same emblematic link: affective processing feels warm.

The semantic relationship highlighted above is likely more than just symbolic. A number of research perspectives, including Barsalou's (1999) framework on perceptual symbol systems, posit that semantic expressions are often underpinned by sensory perception, and cognitions and feelings can elicit perceptual stimulation and somatic responses (Schubert 2005; Barsalou 2008). In other words, the mental accessibility of specific concepts can influence individuals' perceptual states. Pertaining to temperature specifically, this assertion is supported by research findings in neuroscience, which show that the same part of the brain (the insular cortex) is involved in processing both physical and psychological warmth information (Kang et al. 2010). Additional support comes from findings in the psychology and consumer behavior literatures, which has found that angerrelated thoughts (Wilkowski et al. 2009), communion traits (Szymkow et al. 2013), and feelings of loneliness (Zhong and Leonardelli 2008) all have the capacity to influence an individual's temperature perceptions. Extending this logic to the domain of consumer decision-making, it is correspondingly probable that engaging in a semantically warm mental process should also influence temperature perceptions. However, this notion has never been tested empirically.

In the current research, we propose that relying on an affective decision-making process can literally make people feel warmer, and consumers will accordingly compensate for uncomfortable temperatures by relying on affect more or less to warm up or cool down, respectively. Importantly, we argue consumers will rely on affective processing to a greater or lesser degree only if they feel colder or warmer than is comfortable. Such conditions activate thermoregu- latory objectives, which are what ultimately lead to the adoption of a regulatory decision-making style as evidenced by the degree of affective processing. While extant research demonstrated that uncomfortable temperatures can alter consumer preference for materially or metaphorically warm/ cool stimuli (e.g., Hong and Sun 2012; Zhang and Risen 2014), we are the first to suggest that it might systematically change the extent to which consumers rely on affect when making their decisions in the first place. This is highly consequential given that the utilization of affective processing can systematically influence a wide range of consumer responses (e.g., Shiv and Fedorikhin 1999; Hsee and Kunreuther 2000; Hsee and Rottenstreich 2004; Cohen, Pham, and Andrade 2008). Our formal hypotheses can be summarized as follows:

H1: Engaging in affective decision-making will make individuals feel warmer.

H2a: Individuals who feel uncomfortably cold will achieve greater thermal comfort if they adopt an affective decision-making style than if they adopt a cognitive decision-making style.

H2b: However, individuals who feel uncomfortably warm will achieve greater thermal comfort if they adopt a cognitive decision-making style than if they adopt an affective decision-making style.

H3: Individuals will rely more (less) on affect in decision-making when they feel uncomfortably cold (warm).

Together, these hypotheses form our thermoregulatory decision-making framework (see fig. 1): a series of studies that individually support each element of our theorizing and collectively provide compelling evidence for our framework. Study 1 provides initial evidence that reliance on affect in decision-making alters how cold or warm individuals feel (supporting hypothesis 1), and study 2 demonstrates that relying more or less on affect in decision-making can increase temperature-related comfort in response to uncomfortably cold and warm temperatures, respectively (supporting hypotheses $2 \mathrm{a}$ and $2 \mathrm{~b}$ ). The next three studies provide support for hypothesis 3 and our proposed process (fig. 1). Specifically, study 3 demonstrates that individuals instinctively apply this regulatory strategy on their accord, showing that continuously holding on to uncomfortably 


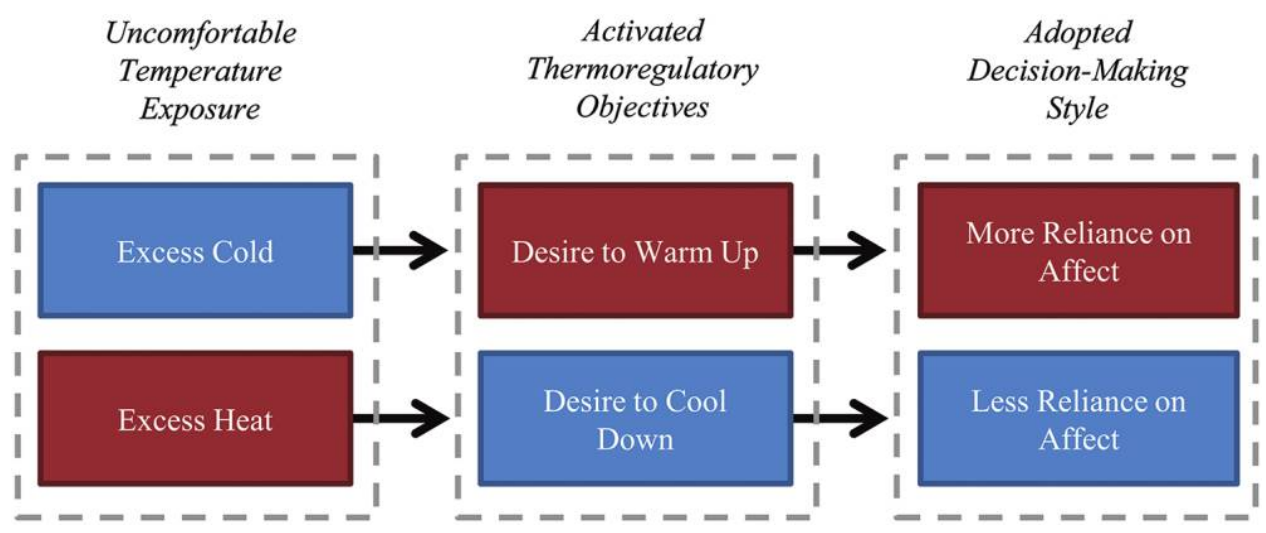

Figure 1. Illustration of our proposed thermoregulatory decision-making framework.

cold (warm) objects leads individuals to spontaneously rely more (less) on affect when assessing a wildlife conservation scenario. Study 4 extends these findings to a different decision-making domain (insurance purchasing) and demonstrates the effect on downstream variable (willingness to pay). Finally, study 5 replicates the effect in ambient temperature conditions and demonstrates that the effects are more exaggerated at extreme temperatures (when thermoregulatory objectives are at their strongest).

\section{STUDY 1: THE WARMING EFFECTS}

\section{OF AFFECTIVE DECISION-MAKING}

Our proposed thermoregulatory decision-making framework is based on hypothesis 1-that the mere use of an affective decision-making process can make people feel warmer. While, as we previously noted, metaphorical links to this effect are prevalent, no empirical evidence exists. To test this hypothesis, participants in this study were given explicit instructions to use either their feelings or evaluative thoughts in assessing a series of advertisements, and we examined the degree to which individuals felt a difference in temperature as a result. We expected that the affective (vs. cognitive) decision-making process would make participants feel warmer (vs. cooler).

Two hundred and one individuals (34\% female, $M_{\text {age }}=$ 33.58, SD $=10.04$ ) recruited from Amazon Mechanical Turk participated in this study in exchange for monetary compensation (the exact breakdown of participants per condition for this study and remaining studies are reported in app. A; apps. A-J are available online). While in all of our subsequent studies we are able to control the exact room temperature settings for participants, in this study we relied on participants' self-reported room temperature to control for ambient temperature differences across partic- ipants' different settings. Accordingly, the recruitment posting instructed participants to only participate if they currently had access to a thermometer or thermostat to read the temperature of the room they were in.

Participants were randomly assigned to one of two conditions before being asked to assess open-endedly a series of print advertisements (the manipulation and procedure were adapted from Pham et al. 2001). In the first condition ("affective instructions"), respondents were instructed to focus on their feelings and emotions when assessing the forthcoming advertisements. In the second condition ("cognitive instructions"), respondents were told to focus on their objective judgments when assessing the advertisements (see app. B for instructions by condition). Afterward, participants indicated their perceived temperature ("Please indicate how warm or cold you currently feel temperaturewise," measured on a 7-point Likert scale anchored by "extremely cold" and "extremely warm"), which was our dependent variable of interest. In addition, participants indicated their mood (on 7-point Likert items: good, cheerful, content, happy, unhappy [reverse coded]; $\alpha=.86$ ) and effort expended in performing the task ("I put a lot of effort into completing this study,") to check whether our manipulation inadvertently led to any differences in these factors. Finally, participants indicated the temperature of their current room (in degrees Fahrenheit), their gender, and their age.

\section{Results and Discussion}

Five participants did not respond to the room temperature question, resulting in 196 observations for analysis. Reported room temperature did not significantly differ across conditions $\left(M_{\text {affective }}=69.50^{\circ} \mathrm{F}, M_{\text {cognitive }}=71.76^{\circ} \mathrm{F} ; p>.16\right)$. As expected, an ANCOVA using the task instructions as the inde- 
pendent variable, perceived temperature as the dependent variable and room temperature as a covariate demonstrated that participants who were given instructions to assess the advertisements using affective judgements reported feeling warmer than those individuals instructed to use cognitive judgements $\left(M_{\text {affective }}=4.78, M_{\text {cognitive }}=4.40 ; F(1,193)=\right.$ $4.21, p=.04$; without the covariate: $p=.03$ ). The instructions manipulation did not have a significant impact on mood or effort expended (both $p>.20$ ).

Results from this study suggest that the mere use of an affective decision-making process can indeed influence how warm individuals feel, supporting hypothesis 1 . Furthermore, the results provide preliminary indication that the perceived temperature differences are not due to mood or effort expended on the task.

\section{STUDY 2: AFFECTIVE DECISION-MAKING CAN INFLUENCE THERMAL COMFORT}

The purpose of study 2 was to examine whether individuals exposed to uncomfortably cold or warm temperatures could achieve some greater level of temperature-related comfort by relying more or less on affect in their decision-making, respectively (hypothesis 2). In this study, individuals were again explicitly instructed to use a particular decision-making style; in our subsequent studies, we examine whether individuals instinctively apply this regulatory strategy on their own accord.

Specifically, we conducted a laboratory experiment in which participants were assigned to either a cold or warm temperature condition, using a cup-holding protocol. After the temperature manipulation, participants were given explicit instructions to describe either their feelings (affective condition) or evaluative thoughts (cognitive condition) in assessing a series of scenarios, and we examined the degree to which they felt thermally comfortable afterward. In accordance with our theorizing, we expected that in the cold condition, participants given the affective task would feel more comfortable than those given the cognitive task, but that in the warm condition, the reverse would be true.

\section{Main Study}

One hundred and seventeen undergraduate students at Baruch College, City University of New York, were assigned to one of four conditions based on a 2 (temperature: cold vs. warm) $\times 2$ (task instructions: affective vs. cognitive) between-subjects design. Participants were asked to hold onto either a cold or warm cup throughout the duration of the experiment with their nondominant hand. As a cover story, respondents were told that the experimenters were interested in their ability to multitask. Rigorous pretesting ensured that this manipulation was externally valid and that warm and cold conditions were uncomfortable enough to provoke thermoregulatory objectives (to cool down and warm up, respectively; see app. $C$ for pretesting procedure and results).

Next, participants were randomly assigned to one of two task conditions to induce either an affective or cognitive decision-making style (adapted from Pham et al. 2001; see app. D for exact wording). The task instructions asked participants to describe either their feelings (affective condition) or evaluative thoughts (cognitive condition) while assessing a series of scenarios (e.g., "going to a rock concert," "taking a test at school," "spending Thanksgiving with your family," and "watching your favorite TV show at home").

Afterward, we asked participants to indicate their temperature-related comfort ("Please indicate how comfortable you currently feel, in terms of temperature") on a 9-point Likert scale anchored by "very uncomfortable" and "very comfortable." Furthermore, some early research suggests that temperature changes may affect arousal levels (Poulton 1976), although literature has documented that uncomfortable temperatures in either direction (warm or cold) are both arousing and sedative (Anderson 1989). Nevertheless, we added measures to assess any differences in perceived arousal (using a 24-item scale; e.g., "alert," "excited," "drowsy," taken from Anderson, Deuser, and DeNeve 1995; $\alpha=.96$ ) or mood (as measured in study $1 ; \alpha=.86$ ) as a result of our temperature manipulation. Finally, participants indicated their age and gender. The entire session lasted approximately 15 minutes.

\section{Results and Discussion}

Thermal Comfort. Because our theorizing suggests that affective decision-making can impact perceived temperatures, we expected an interactive effect of task instructions on thermal comfort, depending on the initially experienced temperature condition. Specifically, we expected that in the cold condition, participants given the affective task would feel more comfortable than those given the cognitive task, but that in the warm condition, the reverse would be true. In other words, because participants in the mixed conditions (cold-affective and warm-cognitive) were able to compensate, we expected them to feel more comfortable than those in the matched conditions (cold-cognitive and warm-affective). Indeed, an ANOVA revealed a significant temperature $\times$ instructions interaction on respondent's thermal comfort 
$(F(1,113)=7.47 ; p<.01)$. Per our theorizing, in the cold condition, participants given the affective instructions were more comfortable than those given the cognitive instructions $\left(M_{\text {affective }}=6.55\right.$ vs. $M_{\text {cognitive }}=5.61, F(1,113)=4.43$, $p<.04)$, but the reverse was true in the warm condition $\left(M_{\text {affective }}=5.79\right.$ vs. $M_{\text {cognitive }}=6.63, F(1,113)=3.14$, $p<.08$; see fig. 2 for a visual representation of the interaction and contrasts). In other words, participants whose instructions had a thermoregulatory tone that contrasted with their initial experienced physical temperature (coldaffective and warm-cognitive conditions) were more comfortable than those whose instructions matched their initial physical temperature manipulation (cold-cognitive and warmaffective conditions). Further, although less central to our hypothesizing, participants with the warm cups felt more comfortable than those with the cold cups within the cognitive instruction condition $(F(1,113)=5.00, p<.03)$, and this pattern was reversed (although not significant) in the affective instructions condition $(F(1,113)=2.68, p=.10)$.

Mood and Arousal. Consistent with our pretest results, an ANOVA demonstrated no significant effects of our temperature or instruction manipulations on mood or perceived arousal (all $p>$.20). Thus, we are able to rule these out as potential alternative explanations.

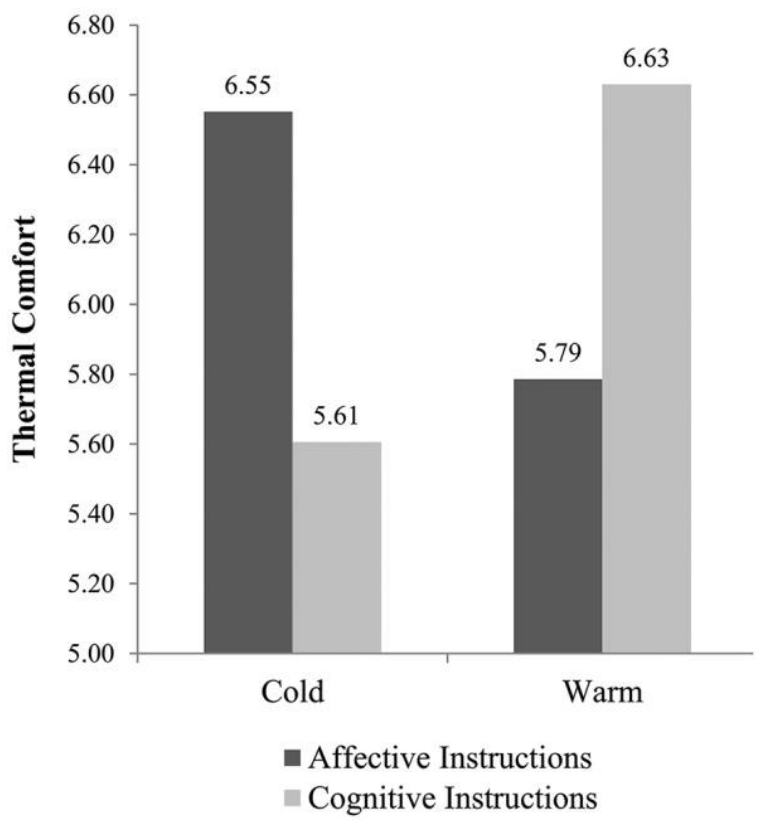

Figure 2. Study 2: In the cold temperature condition, consumers feel more thermal comfort when engaging in an affective task versus a cognitive task. The reverse is true in the warm temperature condition.

\section{STUDY 3: UNCOMFORTABLE TEMPERATURES DRIVE INSTINCTIVE ADOPTION OF DECISION-MAKING STYLES}

Study 2 demonstrated that when individuals are exposed to undesirably cold (warm) temperature conditions, an affective (cognitive) decision-making task could aid in increasing their thermal comfort. The purpose of study 3 was to examine whether individuals would spontaneously adopt this regulatory pattern their own accord, relying more (less) on affect when uncomfortably cold (hot), thus testing hypothesis 3 . To establish a nuanced account of consumer responses to temperature, we examined consumers' reliance on affect along a continuum of temperature conditions. According to our theorizing, we expected the effect to be most exaggerated at extreme temperatures.

\section{Method}

One hundred and sixty-four adults from a community-based subject pool participated in our study in exchange for monetary compensation. Temperature was manipulated using a similar cup-holding procedure as in study 2 . However, as opposed to maintaining two discrete temperature conditions, we instead manipulated temperature across a wide spectrum, including both moderate and more extreme temperatures $\left(38^{\circ} \mathrm{F}-145^{\circ} \mathrm{F}\right.$; the temperature of a cup at room temperature was approximately $70^{\circ} \mathrm{F}$ ). The experimenters used an infrared thermometer to measure and record the exact surface temperature of each cup immediately prior to giving it to each participant.

Participants were presented with a wildlife conservation scenario (describing rescue efforts to save pandas; adapted from Hsee and Rottenstreich 2004, Chang and Pham 2018; see app. E for scenario). Our dependent variable of interest was the extent to which participants relied on affect when assessing the scenario. This was measured via a reliance on affect scale (three items adapted from Shiv and Fedorikhin 1999: When assessing the scenario, I relied on: "my thoughts (1)/my feelings (7)," "my rational side (1)/my emotional side (7)," and "my head (1)/my heart (7)," $\alpha=.83$ ). Consistent with previous literature, we manipulated the number of pandas presented in the scenario (either one or four) as a generalization factor. Given the scenarios were presented in an affect-rich format (pictures of vulnerable pandas), previous literature suggests both conditions provide an opportunity for affective processing, and thus participants should be scope insensitive (Hsee and Rottenstreich 2004; Chang and Pham 2018). Accordingly, we predicted that the colder the temperature, the more participants would rely on affect, irre- 
spective of scope. After collecting a few ancillary measures (reported in app. F) that ensured the participant's task was consistent with the cover story, participants were asked to evaluate their current mood state (as measured in studies 1 and 2; $\alpha=.91$ ) and to indicate their gender and age. The entire session lasted approximately 10 minutes.

\section{Results and Discussion}

Reliance on Affect. One participant did not complete the questionnaire, resulting in 163 observations for analysis. The temperature manipulation did not have a significant impact on mood ( $p>$.70). As expected and confirming hypothesis 3 , a linear regression analysis confirmed a significant main effect of temperature on the reliance on affect scale, holding scope, and the scope $\times$ temperature interaction as covariates $(\beta=-.02, t(159)=-2.63, p<.01$; without covariates: $p<.01)$. The negative coefficient suggests that, as theorized, the colder the temperature, the greater reliance on affect.

Ancillary Analyses. Our theorizing predicts that the colder (warmer) the temperature, the greater (less) the reliance on affect as shown by the regression above. At the same time, the effect of temperature on reliance on affect should be stronger at more extreme (and accordingly more uncomfortable) temperatures at both ends of the temperature spectrum. To assess this, we estimated a cubic regression model, where, starting in the cold temperature range, reliance on affect decreases as temperature increases, then flattens out at neutral temperatures, and then decreases again in the warm temperature range. Results from this analysis showed a significant effect $\left(\beta_{1}=-.16, \beta_{2}=.002, \beta_{3}=\right.$ $.00 ; F(1,159)=3.25, p<.03)$. The negative leading coefficient $\left(\beta_{1}\right)$ supported the expected pattern (only at the high and low ranges of the spectrum did reliance on affect decrease along with increases in temperature).

Confirming hypothesis 3 , results from this study provide initial evidence that individuals instinctively rely more (less) on affect in response to uncomfortable cold (warm) temperature exposure. In the next study, we document the effect such temperature-driven reliance on affect ultimately has on important downstream variable-purchasing decisions.

\section{STUDY 4: DOWNSTREAM EFFECTS OF TEMPERATURE-DRIVEN AFFECTIVE DECISION-MAKING}

While study 3 demonstrated that individuals rely more (less) on affect when exposed to cold (warm) temperature, studies 4 and 5 document the effect of this temperaturedriven processing style on downstream consumer behavior. Specifically, we measured the maximum amount participants would be willing to pay to insure an antique clock (procedure adapted from Hsee and Kunreuther 2000). The clock was given either a high sentiment description (indicating high affective value) or a low sentiment description (indicating low affective value). Previous literature suggests that if one is relying on an affective decision-making process (which we propose should be the case in the cold temperature condition), we should expect participants to be willing to pay more to insure the clock with a high (vs. low) sentiment description. However, if one is not relying on affect (which we propose should be the case in the warm temperature condition), then there should be no difference in willingness to pay across the two object description conditions.

\section{Method}

One hundred and twelve undergraduate students were randomly assigned to one of four conditions according to the 2 (temperature: cold vs. warm) $\times 2$ (object description: low sentiment vs. high sentiment) design. Temperature was manipulated using the same cup-holding procedure as in study 2. After the temperature manipulation, all participants were presented with a hypothetical scenario in which they would have the opportunity to purchase insurance for an antique clock (Hsee and Kunreuther 2000). Participants read a description of the clock which differed depending on condition: the clock was described in either a low sentiment or high sentiment fashion (see app. G for details). In the low sentiment condition, the object description implied no affective value to the subject, while in the high sentiment condition the object description did imply affective value to the subject.

After reading the scenario and object description, respondents indicated the maximum amount they would be willing to pay to insure the clock, which was the main dependent variable of interest. Participants were then asked to indicate the extent to which they relied on affect when making their decision (using the same three-item scale as in study 3; $\alpha=.80$ ). Finally, participants indicated their age and gender. The entire session lasted approximately 10 minutes.

\section{Results and Discussion}

Willingness to Pay for Insurance. As predicted, an ANOVA revealed a significant interaction between temperature and object description on willingness to pay $(F(1,108)=4.46$, $p<.04)$. As expected, in the cold condition, the difference between the low sentiment and high sentiment conditions 
was significant $\left(M_{\text {low sent. }}=\$ 8.71, \quad M_{\text {high sent. }}=\$ 52.12\right.$; $F(1,108)=17.04 p<.001)$, and in the hypothesized direction (participants were willing to pay significantly more for the clock when it had a highly sentimental description). In the warm condition however, as expected, the difference between the two object description conditions was not significantly different $\left(M_{\text {low sent. }}=\$ 19.71, M_{\text {high sent. }}=\$ 32.22\right.$; $F(1,108)=1.52, p>.20)$. Furthermore, the contrast between the cold and warm conditions was not significant in the low sentiment condition $(F(1,108)=1.19 p>.20)$ but was marginally significant in the high sentiment condition $(F(1,108)=3.52 p<.07)$, in that people were willing to pay more to insure the clock when cold (see fig. 3; model specifications are in app. $\mathrm{H}$, panel 1).

Reliance on Affect. As expected and further supporting hypothesis 3, an ANOVA with temperature and object description as independent variables revealed only significant main effects of temperature and object description on participants' reliance on affect $(F(1,108)=3.24, p=.07$ and $F(1,108)=10.26, p<.01$, respectively) but not on the interactive effect of temperature $\times$ object description $(p>.20$; see app. $H$, panel 2). To determine the extent to which reliance on affect mediated the effect of temperature on willingness to pay, we conducted a moderated mediation analysis by applying a three-step series of analyses (following the pro-

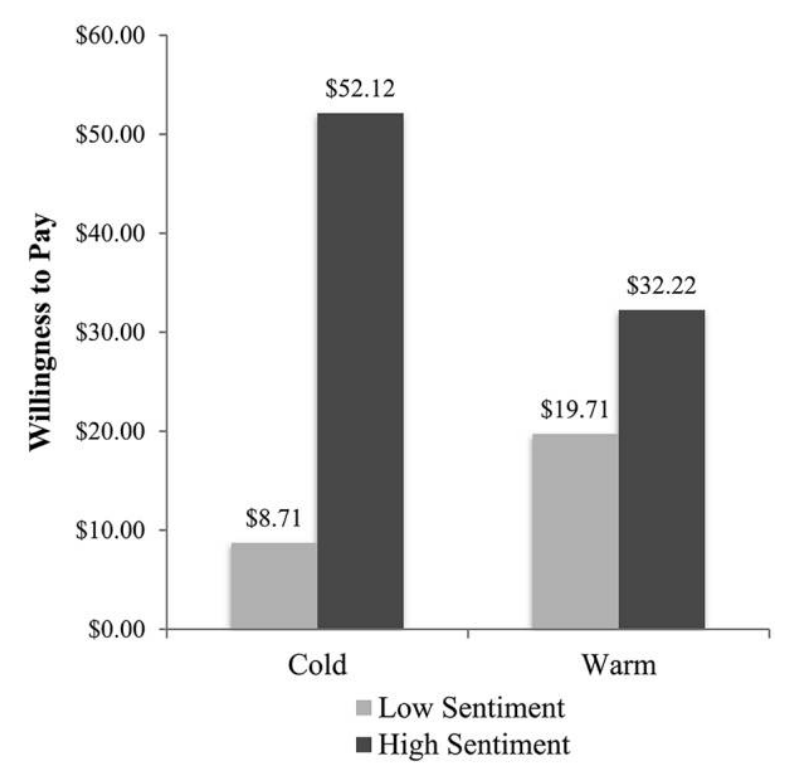

Figure 3. Study 4: In the cold temperature condition, participants were willing to pay significantly more to insure an object when it had high (versus low) sentimental value. In the warm condition they exhibited no difference in willingness to pay for insurance. cedure in Pham et al. 2010, study 1). Specifically, we ran an ANCOVA with temperature and object description as predictors, willingness to pay as the dependent variable, and reliance on affect and its interaction with object description as covariates (see app. H, panel 3). As expected, the interaction between reliance on affect and object description was indeed predictive of overall willingness to pay $(F(1,106)=$ 5.23, $p=.02$, whereas the interaction of temperature by object description is no longer significant $(p=.17)$. This reflects a $64 \%$ reduction in mean square of the temperature by object description interaction produced by controlling for the covariates relative to the model with no covariates included (app. H, panel 1 compared to panel 3; see Pham and Muthukrishnan 2002). Together, results fully support the pattern of moderated mediation as hypothesized.

\section{STUDY 5: EXTENSION TO AMBIENT TEMPERATURE CONDITIONS}

In study 5 we once again examine the downstream consequences of temperature driven processing, but this time extend our temperature manipulation to ambient conditions instead of cup-holding. By employing a wide range of continuous temperature conditions along with precise temperature measurements, this study allowed us to further demonstrate how uncomfortable temperature conditions lead to theoretically different behavioral responses than subtle, comfortable temperature conditions do. In addition, we explicitly test our underlying theoretical assumption that temperatures in the more extreme temperature conditions elicit thermoregulatory objectives. Finally, given that previous research suggests that warm ambient temperatures can hurt consumer performance on complex tasks (Cheema and Patrick 2012), we added measures to assess and control for perceived task difficulty across the different temperature conditions.

\section{Method}

One hundred and ninety-six undergraduate students participated in this study in exchange for monetary compensation. In this study we manipulated both ambient temperature (using air conditioning and heating systems) and object description (low sentiment versus high sentiment). Temperatures included both moderate and more extreme temperatures, ranging from $64^{\circ} \mathrm{F}$ to $91^{\circ} \mathrm{F}$ (all volunteers came from within the same temperature-regulated building, which was approximately $70^{\circ} \mathrm{F}$ ).

In this particular study, participants were seated in a small room, and data was collected one participant at a time 
(since the presence of multiple people could possibly lead to variations in the room temperature). As a cover story, participants were told that before beginning the main study, the experimenter wished to get their opinion about how suitable the experimental environment was. First, as a manipulation check, respondents were asked to indicate how warm or cold they felt on a 7-point scale anchored by "extremely cold" and "extremely warm" and to indicate how comfortable they felt (on 7-point Likert-scale: "Right now I feel comfortable temperature-wise"). Further, to ensure the temperature conditions would induce thermoregulatory objectives, they were asked two additional 7-point Likert-scale items: "I would like the room temperature to be warmer than it is right now," and "I would like the room temperature to be colder than it is right now."

After responding to these introductory questions, all participants were presented with the same hypothetical insurance scenario as in study 4 . Upon reading the scenario, respondents indicated the maximum amount they would be willing to pay for insurance of the clock and were asked to indicate their reliance on affect (as in previous studies, $\alpha=$.83). Finally, participants indicated their gender, age, and how difficult they found the task ("I had difficulty completing this study," on a 7-point Likert scale). The entire session lasted approximately 10 minutes.

\section{Results and Discussion}

Manipulation and Assumption Checks. As mentioned above, we varied temperature along a spectrum and recorded the exact room temperature for each participant. As expected, regression results confirmed a significant positive effect of room temperature on felt temperature (the warmer the temperature, the warmer participants reported feeling: $\beta=.109$, $t(195)=9.35, p<.001)$. Next, we examined the impact of the room temperature manipulation on participants' thermal comfort. We expected a concave U-shaped pattern, where those in the neutral temperature conditions would be the most comfortable temperature-wise, but those participants at the lowest and highest temperatures would be least comfortable temperature-wise. Confirming our theorizing, the test of a quadratic effect was indeed significant, with a negative quadratic term confirming the concave U-shape pattern $\left(F(2,192)=4.72, \beta_{2}=-.010, p<.02\right)$. Additional regression results confirmed that temperature had a significant negative effect on participants' desire for the room to be warmer $(\beta=-.147, t(195)=-7.27, p<.001)$ and a significant positive effect on their desire for the room to be colder $(\beta=.191, t(195)=10.16, p<.001)$. These findings confirm our underlying theoretical assumption that cold and hot temperatures induce thermoregulatory objectives to warm up and cool down, respectively.

Willingness to Pay for Insurance. One participant did not respond to the willingness to pay question, resulting in 195 observations for analysis. A regression analysis with temperature, object description, and their interaction as predictors and willingness to pay as the dependent variable revealed a significant interaction of temperature $\times$ object description on willingness to pay $(\beta=-1.29, t=-2.09$, $p<.04)$. This indicates that the difference in willingness to pay between the two object description conditions varied significantly across the temperature conditions. A simple slopes analysis mirrored the results found in study 4: in the colder temperatures ( -1 SD below the mean), the difference between the low sentiment and high sentiment conditions was significant $\left(M_{\text {lowsent. }}=\$ 17.54, M_{\text {high sent. }}=\right.$ $\$ 61.67 ; \beta=44.14, t=4.26, p<.001)$ and in the hypothesized direction (participants were willing to pay significantly more for the clock when it had a highly sentimental description). In warmer temperature conditions ( +1 SD above the mean), the difference between the two object description conditions was not significantly different $\left(M_{\text {low sent. }}=\right.$ $\left.\$ 24.34, M_{\text {high sent. }}=\$ 37.75 ; \beta=13.42, t=1.29, p>.19\right)$.

Importantly, the continuous measurement of temperature along a continuum allowed us to define meaningful boundaries for when we would expect particular behavioral responses. Thus, we applied the Johnson and Neyman (1936) technique, to identify regions of the temperature continuum where the effect of object description on willingness to pay is significant and where it is not. Results indicated that once temperatures reached approximately $78^{\circ} \mathrm{F}$ and above (representing an approximate $8^{\circ} \mathrm{F}$ increase above the baseline room temperature), participants no longer show any difference of willingness to pay as a result of the differing object descriptions, suggesting that those participants were not relying on an affect in making their decisions (see app. I for the complete Johnson and Neyman analysis results, with the cutoff point highlighted). In fact, at the warmest temperature, the object description is completely irrelevant to subjects- suggesting complete absence of affect in decision-making $(\beta=-15.43, t=-.69, p>.40)$, while at the coldest temperature, the effect of object description is the largest, indicating the most affective decision-making $(\beta=54.36, t=3.82, p<.001)$. This is once again theoretically consistent with our goal-motivated regulatory account, in that more extreme temperatures should be more likely to 
trigger thermoregulatory objectives and provoke contrasting decision-making accordingly.

Reliance on Affect. To confirm the predicted pattern of moderated mediation, we applied a three-step series of regression analyses (see app. J for model specifications). Based on the results of the Johnson and Neyman analysis above, we examined how temperatures above and below $78^{\circ} \mathrm{F}$ would differentially effect participants' decision-making (a regression analysis using this temperature distinction replicated the interactive effect of temperature $\times$ object description on willingness to pay; $\beta=-7.43, t=-1.72, p=.087$; see app. J, panel 1). As expected, a regression analysis with temperature, object description, and their interaction as predictors and reliance on affect as the dependent variable (see app. J, panel 2) revealed significant main effects of both temperature and object description on participants' reliance on affect in the predicted directions $(\beta=-.257, t=-1.81$, $p=.07$ and $\beta=.394, t=2.77, p<.01$, respectively) and a nonsignificant effect of temperature $\times$ object description $(p>.50)$. We then ran a regression analysis with willingness to pay as the dependent variable with temperature, object description, temperature $\times$ object description, reliance on affect, and reliance on affect $\times$ object description as predictors (see app. J, panel 3). As anticipated, the interaction between reliance on affect and object description is significant $(\beta=$ $6.83, t=3.33, p<.01$ ), whereas the interaction of temperature by object description is no longer significant $(\beta=$ $-4.79, t=-.09, p=.239)$. This reflects a $59 \%$ reduction in mean square of the temperature by object description interaction produced by controlling for the covariates relative to the model with no covariates included (see Pham and Muthukrishnan 2002). Consistent with study 4 and with our theorizing, results confirm a pattern of moderated mediation. Collectively, results from this study are convergent with those of studies 3 and 4 (in support of hypothesis 3), suggesting that the impact of tactilely induced and atmospherically induced temperature changes operate similarly in their impact on an individual's reliance on affect in decision-making, and this impact is most pronounced at more extreme (and hence more uncomfortable) temperatures.

Ancillary Analyses. As mentioned previously, research suggests that warm ambient temperatures can be more depleting than cool temperatures (Cheema and Patrick 2012). Linear regression results demonstrated a significant effect of temperature on perceived task difficulty (the warmer the temperature, the greater task difficulty participants re- ported experiencing: $\beta=.03, t(193)=1.99, p<.05)$, replicating the effect found in previous research (Cheema and Patrick 2012). While we did not expect this to explain the pattern of our results (Cheema and Patrick predict that warm temperature should only limit consumers' ability to perform complex tasks, and the task in the current experiment is relatively simple, leaving no reason to expect reliance on affect to result in more or less task difficulty), we nevertheless assessed whether task difficulty might be a competing explanation for our findings. Importantly, task difficulty was not a significant predictor of willingness to pay or reliance on affect (both $p>.40$ ). Furthermore, we reran the analyses above, controlling for perceived task difficulty, and this did not change our results (the resulting interaction of temperature $\times$ object description on willingness to pay remained significant: $(\beta=-2.56, t=-2.06$, $p<.05$; and the contrasts and mediation analyses results continue to hold respectively). Overall, this final study replicates the thermoregulatory pattern found in studies 3 and 4 , but this time it extends the results to ambient temperatures.

\section{GENERAL DISCUSSION}

Collectively, results from five studies support our proposed thermoregulatory decision-making framework (fig. 1). Study 1 demonstrates the warming property of affective decisionmaking, and study 2 shows that the adoption of an affective (cognitive) pathway can improve temperature-related comfort in response to uncomfortable cold (warmth). Studies 3-5 demonstrate that individuals instinctively apply this regulatory strategy on their accord, spontaneously relying more (less) on affect when feeling uncomfortably cold (warm). We demonstrate the effect holds in response to both tactile and ambient temperature manipulations and across multiple decision-making contexts and show the downstream effects on willingness to pay. Finally, by looking at a wide continuum of temperatures, we demonstrate that the effect is most exaggerated at extreme temperatures (when thermoregulatory objectives are at their strongest).

This research contributes to previous literature examining the effect of cold temperature on consumer responses. Our finding that cold can in fact alter consumers' reliance on affect in making decisions may help explain previous findings (e.g., affective processing should boost preference for affectively superior stimuli), but our work also broadens the scope of downstream effects that may stem from uncomfortable temperature exposure, given the numerous domains in which affective decision-making has been shown to play a 
formative role (e.g., financial, charitable, and self-regulatory domains; e.g., Shiv and Fedorikhin 1999; Hsee and Kunreuther 2000; Hsee and Rottenstreich 2004; Cohen et al. 2008).

We also lend nuance to the study of atmospherics and temperature effects in particular by demonstrating the differential effects of modest, comfortable temperatures versus more exaggerated temperatures (by manipulating temperature on a continuum in studies 3 and 5). While we replicate Cheema and Patrick's (2012) finding that increased temperatures can be depleting, we also find that at uncomfortable temperature levels, activated thermoregulatory goals can elicit contrasting decision-making styles.

Our work can also be considered vis-a-vis affective decisionmaking literature from two separate angles. First, there have been recent calls for research exploring antecedents to affective processing, or "affective engineering" (Cohen et al. 2008; Pham 2014). The current research represents a step in this direction by proposing that uncomfortably cold temperature exposure may be one such precursor to increased use of affective information in decision-making.

Second, scholars have often emphasized that reliance on affect should not be deemed irrational, as affective processing has certain systematic advantages over more cognitive processing (e.g., decisions are made more quickly, and are more stable and consistent across individuals; Pham et al. 2001; Pham 2007). Our research uncovers an additional potential benefit that stems from reliance on affect: feelings of warmth. While this sensation alone offers immediate value in uncomfortably cold conditions (consistent with research suggesting the affective-system is present-oriented, Chang and Pham 2013), there may be longer-term implications. For example, given that reliance on emotions has generally been shown to promote more social behavior (Pham 2007) and foster interpersonal bonding (Spoor and Kelly 2004), affective processing may function as an inadvertent way to promote more successful social interactions during times of interpersonal isolation (which can feel both subjectively and objectively cold; Ijzerman et al. 2012). Future research might examine this notion and potentially uncover additional regulatory benefits that stem from affective processing.

The current work has a number of interesting implications for industry practitioners and public policy makers. Given that reliance on affect tends to increase the value consumers assign to hedonic (vs. utilitarian) product attributes (e.g., Shiv and Fedorikhin 1999; Dhar and Wertenbroch 2000), retailers offering hedonic, luxury goods and services would likely benefit from consumers' affective processing and thus may profit by exposing shoppers to cold temperatures in the marketplace (consistent with examples in this article's introduction). Interestingly, cold settings may also elicit affective processing from nonconsumers - that is, store employees. For example, research suggests that colder store temperatures induce service employees to give customers greater discounts, even when doing so results in reduced commission for themselves (Kolb, Gockel, and Werth 2012). This finding is consistent with our theorizing, as such behavior is likely to result from affective interpersonal assessments.

Furthermore, aside from temperature manipulation via store or service-based thermostat control, companies can impact individuals' experienced temperature via nontraditional promotional campaigns. For example, Kraft built and maintained heated bus shelters in an attempt to convey the warmth that consumers would feel from eating stuffing (Elliott 2008). While such promotional campaigns are meant to highlight product benefits, companies should be cognizant of potential unintended consequences on a consumer's decision-making style should the temperatures reach uncomfortable levels.

It is also interesting to consider how the current findings might provide insight to public policy makers concerned with consumers' financial well-being. Given that reliance on affect can impair financial decision-making (e.g., leading to objectively irrational insurance purchasing decisions; Hsee and Kunreuther 2000; Chang and Pham 2018), overly cold temperature settings inside retail banks, mortgage firms, and insurance agencies could possibly exert detrimental effects on consumers' financial choices. Further, since affective judgements can bias individuals' assessments of risk (e.g., Finucane et al. 2000), cold temperatures might also influence gambling behaviors in places like casinos (which are commonly known to maintain a very low thermostat setting; Best of Vegas 2008). Given the consequential nature of these domains, those concerned with protecting consumer welfare might consider mandating a minimum indoor temperature in such institutions, or as a less regulatory measure, alerting consumers to "bundle up" in such venues to avoid feeling uncomfortably cold when making consequential decisions.

Despite the above-mentioned concerns, reliance on affect has also been shown to improve consumer outcomes in many contexts (Pham 2007), and thus cold temperatures hardly pose an unwavering liability to consumers. For example, cinemas are known to maintain chilly interiors (Flynn 2015), and it may be that such cold temperatures induce more affective processing of the films being played, enhancing a viewer's enjoyment of emotionally laden content. This 
logic further implies that cold temperature settings would increase consumer's appreciation of other experiential content high affective value (e.g., opera, live theatre, sporting events). Future research might test these interesting predictions both in the lab and in the field.

Given the wide range of atmospheric and product-based sensations consumers are exposed to everyday, we hope the current work motivates additional research to build a more comprehensive account for when, why, and how such variables can systematically influence consumer decision-making in various consequential domains.

\section{REFERENCES}

Anderson, Craig A. (1989), "Temperature and Aggression: Ubiquitous Effects of Heat on Occurrence of Human Violence," Psvchological Bulletin, 106 (1), 74-96.

Anderson, Craig A., William E. Deuser, and Kristina M. DeNeve (1995), "Hot Temperatures, Hostile Affect, Hostile Cognition, and Arousal: Tests of a General Model of Affective Aggression," Personality and Social Psychologv Bulletin, 21 (5), 434-48.

Barsalou, Lawrence W. (1999), "Perceptual Symbol Systems," Behavioral and Brain Sciences, 22 (4), 577-609.

- (2008), "Grounded Cognition," Annual Review of Psychology, 59, $617-45$.

Best of Vegas (2008), "How to Look Cool While Staying Cool in Las Vegas," retrieved from https://www.bestofvegas.com.

Bruno, Pascal, Valentyna Melnyk, and Franziska Völckner (2016), “Temperature and Emotions: Effects of Physical Temperature on Responses to Emotional Advertising," International Journal of Research in Marketing, 34 (1), 302-20.

Chang, Hannah H., and Michel Tuan Pham (2013), "Affect as a DecisionMaking System of the Present," Journal of Consumer Research, 40 (1), $42-63$.

_ (2018), "Affective Boundaries of Scope Insensitivity," Journal of Consumer Research, 45 (2), 403-28.

Cheema, Amar, and Vanessa M. Patrick (2012), "Influence of Warm Versus Cool Temperatures on Consumer Choice: A Resource Depletion Account," Journal of Marketing Research, 49 (6), 984-95.

Cohen, Joel, Michel Pham, and Eduardo Andrade (2008), "The Nature and Role of Affect in Consumer Behavior," In Handbook of Consumer Psychology, ed. Curtis P. Haugtvedt, Paul M. Herr, and Frank R. Kardes, Mahwah, NJ: Lawrence Erlbaum, 297-348.

Dhar, Ravi, and Klaus Wertenbroch (2000), "Consumer Choice Between Hedonic and Utilitarian Goods," Journal of Marketing Research, 37 (1), 60-71.

Elliott, Stuart (2008), "Hot Food, and Air, at Bus Stops," New York Times, December 2.

Evans, Jonathan St. B. T. (2008), "Dual-Processing Accounts of Reasoning, Judgment, and Social Cognition," Annual Review of Psychology, 59, 255-78.

Feldman Barrett, Lisa, Kevin N. Ochsner, and James J. Gross (2007), "On the Automaticity of Emotion," In Social Psychology and the Unconscious: The Automaticity of Higher Mental Processes. Frontiers of Social Psychology, ed. John A. Bargh, New York: Psychology Press, 173-217.
Finucane, Melissa L., Ali Alhakami, Paul Slovic, and Stephen M. Johnson (2000), "The Affect Heuristic in Judgments of Risks and Benefits," Journal of Behavioral Decision Making, 13 (1), 1-17.

Flynn, Caitlin (2015), "Why Are Movie Theaters Cold? 4 Reasons Summer Blockbuster Season Can Leave You Shivering," Bustle, July 23.

Förster, Jens, Nira Liberman, and Ronald S. Friedman (2007), "Seven Principles of Goal Activation: A Systematic Approach to Distinguishing Goal Priming from Priming of Non-Goal Constructs," Personality and Social Psychology Review, 11 (3), 211-33.

Gross, Charles G. (1995), "Aristotle on the Brain," The Neuroscientist, 1 (4), $245-50$.

Hong, Jiewen, and Yacheng Sun (2012), "Warm It Up with Love: The Effect of Physical Coldness on Liking of Romance Movies," Journal of Consumer Research, 39, 293-306.

Hsee, Christopher K., and Howard C. Kunreuther (2000), "The Affection Effect in Insurance Decisions," Journal of Risk and Uncertaintv, 20, 141-59.

Hsee, Christopher K., and Yuval Rottenstreich (2004), "Music, Pandas, and Muggers: On the Affective Psychology of Value," Journal of Experimental Psvchologv: General, 133 (1), 23.

Ijzerman, Hans, Marcello Gallucci, Wim T. J. L. Pouw, Sophia C. Weißgerber, Niels J. Van Doesum, and Kipling D. Williams (2012), "Cold-Blooded Loneliness: Social Exclusion Leads to Lower Skin Temperatures," $\underline{\text { Acta }}$ Psvchologica, 140 (3), 283-88.

Ijzerman, Hans, and Gün R. Semin (2009), "The Thermometer of Social Relations Mapping Social Proximity on Temperature," $\underline{\text { sychological Sci- }}$ ence, 20 (10), 1214-20.

Johnson, Palmer Oliver, and Jerzy Neyman (1936), "Tests of Certain Linear Hypotheses and Their Applications to Some Educational Problems," Statistical Research Memoirs, 1, 57-93.

Kang, Yoona, Lawrence E. Williams, Margaret S. Clark, Jeremy R. Gray, and John A. Bargh (2010), "Physical Temperature Effects on Trust Behavior: The Role of Insula," Social Cognitive and Affective Neuroscience, 6 (4), 507-15.

Keren, Gideon, and Yaacov Schul (2009), "Two Is Not Always Better than One: A Critical Evaluation of Two-System Theories," Perspectives on Psychological Science, 4 (6), 533-50.

Kolb, Peter, Christine Gockel, and Lioba Werth (2012), "The Effects of Temperature on Service Employees' Customer Orientation: An Experimental Approach," Ergonomics, 55 (6), 621-35.

Lakoff, George, and Mark Johnson (1999), Philosophy in the Flesh: The Embodied Mind and Its Challenge to Western Thought, New York: Harper-Collins.

Lee, Seung Hwan Mark, Jeff D. Rotman, and Andrew W. Perkins (2014), "Embodied Cognition and Social Consumption: Self-regulating Temperature through Social Products and Behaviors," Journal of Consumer Psychology, 24 (2), 234-40.

Metcalfe, Janet, and Walter Mischel (1999), "A Hot/Cool System Analysis of Delay of Gratification: Dynamics of Willpower," Psychological Review, 106, 3-19.

Newman, Andrew (2011), "Outerwear's Spokesman Does without Outerwear," New York Times, 3 November.

Panksepp, Jaak (1998), Affective Neuroscience: The Foundations of Human and Animal Emotions, New York: Oxford University Press.

Peters, Ellen, Daniel Västfjäll, Tommy Gärling, and Paul Slovic (2006), "Affect and Decision Making: A 'Hot' Topic," Journal of Behavioral Decision Making, 19 (2), 79.

Pham, Michel Tuan (2007), "Emotion and Rationality: A Critical Review and Interpretation of Empirical Evidence," Review of General Psychology, $11(2), 155$. 
(2014), "Feelings and Emotions in Consumer Behavior: Future Directions," 49th AMA-Sheth Foundation Doctoral Consortium, Evanston, Illinois.

Pham, Michel Tuan, Joel B. Cohen, John W. Pracejus, and G. David Hughes (2001), "Affect Monitoring and the Primacy of Feelings in Judgment," Journal of Consumer Research, 28 (2), 167-88.

Pham, Michel Tuan, Caroline Goukens, Donald R. Lehmann, and Jennifer Ames Stuart (2010), "Shaping customer satisfaction through selfawareness cues," Journal of Marketing Research, 47 (5), 920-32.

Pham, Michel Tuan, and A. V. Muthukrishnan (2002), "Search and Alignment in Judgment Revision: Implications for Brand Positioning," Journal of Marketing Research, 39, 18-30.

Poulton, E. Christopher (1976), “Arousing Environmental Stresses can Improve Performance, Whatever People Say," Aviation, Space, and Environmental Medicine, 47, 1193-204.

Pursglove, Anna (2013), "Brrr! Why Your Supermarket Is CHILLIER than the Arctic," Daily Mail, http://www.dailymail.co.uk/.

Salkin, Allen (2005), "Shivering for Luxury," New York Times, 26 June.

Schubert, Thomas W. (2005), "Your Highness: Vertical positions as Perceptual Symbols of Power," Journal of Personality and Social Psychologv, 89, 1-21.

Sela, Aner, and Baba Shiv (2009), "Unraveling Priming: When Does the Same Prime Activate a Goal versus a Trait?” Journal of Consumer Research, 36 (3), 418-33.

Shiv, Baba, and Alexander Fedorikhin (1999), "Heart and Mind in Conflict: The Interplay of Affects and Cognition in Consumer Decision Making," Journal of Consumer Research, 26 (3), 278-92.

Spoor, Jennifer R., and Janice R. Kelly (2004), “The Evolutionary Significance of Affect in Groups: Communication and Group Bonding," Group Processes and Intergroup Relations, 7 (4), 398-412.
Szymkow, Aleksandra, Jesse Chandler, Hans Ijzerman, Michal Parzuchowski, and Bogdan Wojciszke (2013), "Warmer Hearts, Warmer Rooms: How Positive Communal Traits Increase Estimates of Ambient Temperature," Social Psychology, 44 (2), 167-76.

Taufik, Danny, Jan Willem Bolderdijk, and Linda Steg (2014), “Acting Green Elicits a Literal Warm Glow,” Nature Climate Change, 5 (1), 37-40.

Wilkowski, Benjamin M., Brian P. Meier, Michael D. Robinson, Margaret S. Carter, and Roger Feltman (2009), "Hot-Headed Is More than an Expression: The Embodied Representation of Anger in Terms of Heat," Emotion, 9 (7), 464-77.

Willet, Megan (2015), “The Ridiculous Reason Why Hong Kong Malls Are Extremely Cold," Business Insider UK, http://uk.businessinsider.com/.

Williams, Lawrence E., and John A. Bargh (2008), "Experiencing Physical Warmth Promotes Interpersonal Warmth,” Science, 322 (5901), 606-7.

Zhang, Meng, and Xiuping Li (2012), "From physical weight to psychological significance: The contribution of semantic activations," Journal of Consumer Research, 38 (6), 1063-75.

Zhang, Yan, and Jane L. Risen (2014), “Embodied Motivation: Using a Goal Systems Framework to Understand the Preference for Social and Physical Warmth," Journal of Personality and Social Psychology, 107 (6), 965-77.

Zhong, Chen-Bo, and Geoffrey J. Leonardelli (2008), "Cold and Lonely: Does Social Exclusion Literally Feel Cold?” Psychological Science, 19 (9), 838-42.

Zhou, Xinyue, Tim Wildschut, Constantine Sedikides, Xiaoxi Chen, and A. J. Vingerhoets (2012), "Heartwarming Memories: Nostalgia Maintains Physiological Comfort," Emotion, 12 (4), 678-84.

Zwebner, Yonat, Leonard Lee, and Jacob Goldenberg (2013), "The Temperature Premium: Warm Temperatures Increase Product Valuation," Journal of Consumer Psychology, 24 (2), 251-59. 